\title{
Nivel de satisfacción en el aprendizaje del idioma inglés en un entorno totalmente digital por tiempos de Covid-19
}

DOI: https://doi.org/10.33262/ap.v3i3.1.81

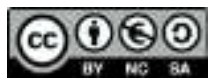

Satisfaction level of English language learning in a fully digital environment by Covid-19 times

Leonardo Efraín Cabezas Arévalo. ${ }^{1}$

\begin{abstract}
.
Introduction: The pandemic has brought face-to-face classes to a completely virtual environment, which has brought distinct advantages and disadvantages for students. For this reason, students have different levels of satisfaction in the development of their classes. Since these are indicators that allow continuous improvement, we should implement frequently questionnaires to know the satisfaction status of the students. Aim: To identify the satisfaction levels of the students of the Escuela Superior Politécnica de Chimborazo in English as a foreign language class in an online environment. Method: 124 students took part in the satisfaction survey with 9 questions. We ordered the options from 1 to 5 where 1 corresponded to: "very dissatisfied", 2: "dissatisfied", 3: "neither satisfied nor dissatisfied", 4: "satisfied" and 5: "very satisfied". Microsoft Form software was used to prepare the survey, and they disseminated it through Microsoft Teams. Results: In the questionnaires, there were preferences in the choice of option 4 "satisfied" which shows high levels of satisfaction in most of the students, while option 2 "dissatisfied" was the least chosen, thus identifying the low levels of student dissatisfaction. Conclusion: Satisfaction levels were high for all questions, which
\end{abstract}

\footnotetext{
${ }^{1}$ Escuela Superior Politécnica de Chimborazo, Administración de Empresas. Chimborazo. leonardoe.cabezas@espoch.edu.ec
} 
enhances virtual education and merges it as an educational alternative for teaching and learning English as a foreign language.

Keywords: connectivity, virtual education, English, covid-19, satisfaction, satisfaction.

\section{Resumen.}

Introducción: La pandemia acarreo que las clases presenciales se desarrollen en un entorno completamente virtual lo que ha traído diferentes ventajas y desventajas para los estudiantes. Por este motivo los estudiantes tienen diferentes niveles de satisfacción en el desarrollo de sus clases. Ya que estos son indicadores que permiten el mejoramiento continuo se deben implementar cuestionarios con frecuencia para conocer el estatus de satisfacción los estudiantes. Objetivo: Identificar los niveles de satisfacción de los estudiantes de la Escuela Superior Politécnica de Chimborazo en clases de inglés como lengua extranjera en un entorno Online. Metodología: 124 estudiantes participaron en la encuesta de satisfacción que contaba con 9 preguntas en total. Las opciones fueron ordenadas del 1 al 5 en donde 1 correspondía a: "muy insatisfecho", 2: "insatisfecho", 3: "ni satisfecho ni insatisfecho", 4: "satisfecho" y 5 "muy satisfecho". Se empleó el software Microsoft Form para la elaboración de la encuesta y se difundió a través de la Microsoft Teams. Resultados: En los cuestionamientos existieron preferencias en la elección de la opción 4 "satisfecho" lo que demuestra niveles altos de satisfacción en la mayoría de los estudiantes mientras que la opción 2 "insatisfecho" fue la menos escogida identificando de esta manera los bajos índices de insatisfacción del estudiante. Conclusión: Los niveles de satisfacción fueron altos para todas las interrogantes lo cual enaltece la educación virtual y se consolida como una alternativa educativa para la enseñanza y aprendizaje del idioma inglés como lengua extranjera.

Palabras claves: conectividad, Educación virtual, inglés, covid-19, satisfacción

\section{Introducción.}

El contexto actual impuesto por la Covid-19 y la transformación de esta enfermedad pandemia ha afectado de una manera directa en la forma en la que vivimos, nos comunicamos y aprendemos (Prodanović \& Gavranović, 2021).

En Ecuador, el 16 de marzo por parte del Estado se imponen diferentes medidas para mitigar el alto índice de contagios que en esos momentos ponían en "jaque" a los servicios sanitarios. Estas medidas implementaban una restricción de movilidad, cerrar negocios y evitar actividades no esenciales. (Santana-Sardi et al., 2020)

Los gobiernos al tomar estas medidas obligan al cierre indefinido de instituciones educativas para evitar la propagación del virus. Estas entidades públicas y privadas en todos sus niveles optan por una formación y enseñanza por medio de las tecnologías de 
la información y comunicación (TIC) como su herramienta principal transformando una educación tradicional a una totalmente virtual. (Flores et al., 2020)

El nuevo ambiente educacional que se instaura provoca mucho debate y se transforma en un cuerpo de estudio en diferentes aspectos, en cuanto a sus ventajas, desventajas y retos que se imponen al tener un contexto totalmente virtual.

Las clases en un entorno digital recurren a herramientas tecnológicas que facilitan el proceso enseñanza-aprendizaje. Banda ancha, audio, video, medios digitales (computadora, Smartphone, etc.), blogs, aulas virtuales son herramientas implementadas para el desarrollo diaria de clases en la actualidad. (Garcia Bejarano et al., 2013)

Aunque estos tipos de herramientas se las venía utilizando de forma usual no eran una pieza central y eje fundamental para la educación de tercer nivel. El cambio en el protagonismo de estas herramientas ha causado una mudanza de hábitos en el estudiante e incluso de los docentes los cuales tenían un papel fundamental en el proceso de enseñanza aprendizaje ahora han dado la posta para que los estudiantes sean ellos mismo el eje central de su propio camino de aprendizaje ya que el docente queda en un segundo plano solamente como guía, orientador y ejemplo. (Cabezas et al., 2021; Monasterio \& Briceño, 2020)

No todos los estudiantes poseen las mismas herramientas digitales y se aprecia que en muchos de los casos su conectividad y enlace a fuentes de internet no son las ideales lo que compromete su entorno de aprendizaje y con ellos sus niveles de satisfacción pueden variar lo que se refleja en las evaluaciones semestrales y estándares de satisfacción. (Cabezas et al., 2021)

La enseñanza del idioma inglés no es ajena a esta tendencia ya que en la actualidad existe un entorno totalmente digital con el implemento de interfaces que utilizan medios de conexión a internet lo que permite tener un ambiente de aprendizaje mucho más visual y auditivo que ayuda a generar una familiaridad para los estudiantes. (Cabezas et al., 2021).

El aprendizaje del idioma inglés no se lo considera como un privilegio en la actualidad sino más bien como un derecho infaltable en la educación lo que permite crear nuevas aptitudes en el estudiante que reforzara sus herramientas para su futuro personal y académico (Cabezas et al., 2019). Este cambio de paradigma a permitido que más personas puedan evidenciar las nuevas oportunidades y puertas que se abren al poder entender y comunicarse a través de un idioma totalmente diferente al suyo. (Cabezas et al., 2021)

Los niveles de satisfacción de los y las estudiantes son un indicativo de la calidad de educación que ellos reciben. Estos indicativos nos guían a establecer bases clara para el mantenimiento, cambio y/o mejoramiento de las prácticas impartidas por los profesores e instituciones educativas.(Zambrano R., 2016) 
A pesar de ser un indicador de gran importancia para el mejoramiento y aplicación de nuevas estrategias en el proceso enseñanza aprendizaje este no se ha estudiado en profundidad en el sector educativo ecuatoriano en tiempos de pandemia. (Alonso, 2010)

Por lo antes expuesto el objetivo de esta investigación fue la de identificar los niveles de satisfacción de los estudiantes en el aprendizaje del idioma inglés como lengua extranjera de la Escuela Politécnica de Chimborazo en un entorno online comparada con un entorno de clases presenciales.

\section{Metodología.}

\section{Población de estudio}

124 estudiantes de la Escuela Superior Politécnica de Chimborazo (ESPOCH) participaron en el desarrollo del estudio. Se seleccionaron a los alumnos de las carreras de Transporte y Administración de Empresas de 3er y 4to nivel de la asignatura de inglés en el período 2021-2021.

\section{Criterios de inclusión}

Alumnos de la ESPOCH que se encuentren en el 3er y 4to nivel de la asignatura de inglés en el período 2021-2021 correspondientes a Transporte y Administración de Empresas.

\section{Criterios de exclusión}

Alumnos de la ESPOCH que no se encuentren en el 3er y 4to nivel de la asignatura de inglés en otro período no correspondiente al año 2021-2021 que no pertenezcan a Transporte y Administración de Empresas.

\section{Diseño del estudio}

Este estudio evalúo los niveles de satisfacción en la enseñanza y aprendizaje online de la asignatura de inglés como lengua extranjera en un periodo aproximado de 6 meses. Las clases se impartieron por medio de la plataforma Microsoft Teams durante el periodo 2021-2021 por un mismo docente. El carácter del estudio fue observacional y transversal, la obtención de los datos de estudio se los extrajo de una encuesta descriptiva de preguntas cerradas. Las opciones de respuesta estuvieron calcificadas del 1 al 5 en donde 1 correspondía a: "muy insatisfecho", 2: "insatisfecho", 3: "ni satisfecho ni insatisfecho", 4: "satisfecho" y 5 "muy satisfecho". Las preguntas se relacionan a los niveles de satisfacción del estudiante basados en la encuesta realizada por Prodanovic (Prodanović \& Gavranović, 2021). Se utilizó la plataforma Microsoft Form para difundir la encuesta por medio de la Plataforma Microsoft Teams a la que los estudiantes tenían acceso y que pudieran desarrollar sin inconvenientes.

\section{Análisis Estadístico}

El análisis estadístico descriptivo se llevó a través del software Microsoft Excel detallando la cantidad de estudiantes que respondieron la encuesta y ordenan las 
respuestas según el número de elección entre los alumnos al igual que los porcentajes finales para cada una de las interrogantes.

\section{Resultados.}

Los preguntas y resultados se encuentran detalladas en las tablas 1-9.

Tabla1. Pregunta 1 ¿Qué tan satisfecho está con la disponibilidad de material de aprendizaje (libros, blogs, audios, videos)?

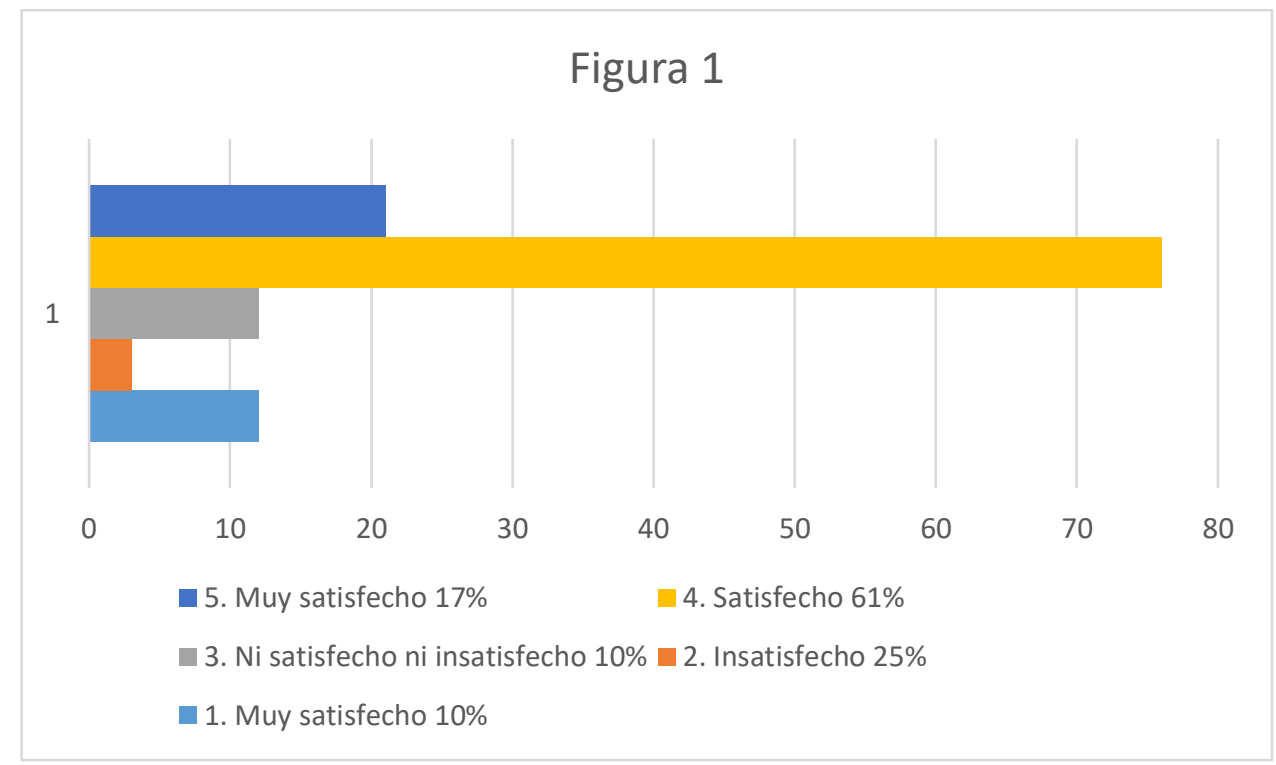

Fuente: Encuesta divulgada por Microsoft Form (Cabezas et al., 2021)

Tabla 2. Pregunta 2 ¿Qué tan satisfecho está con la dinámica de aprendizaje-enseñanza (plataformas de enseñanza eje. Microsoft Teams, aula virtual Moodle?

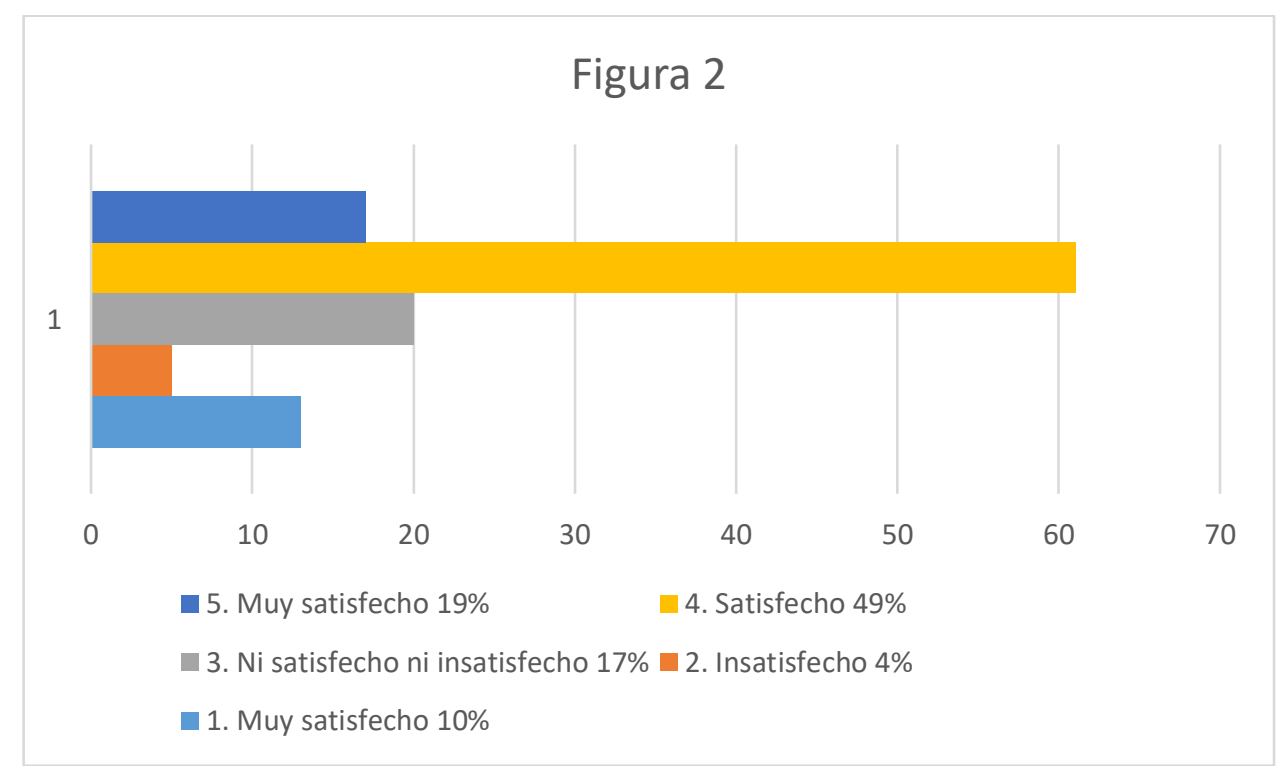

Fuente: Encuesta divulgada por Microsoft Form (Cabezas et al., 2021) 
Tabla 3. Pregunta 3 ¿Qué tan satisfecho está con la comunicación entre docente y estudiantes?

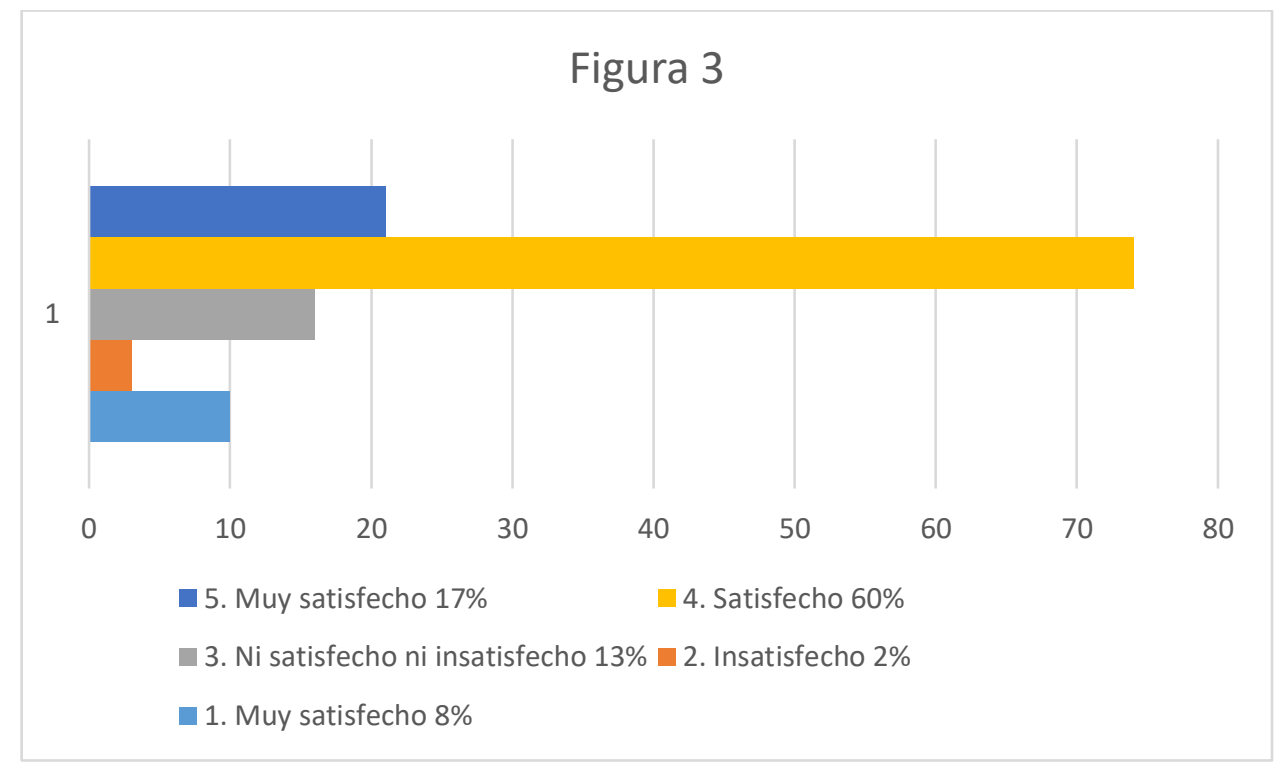

Fuente: Encuesta divulgada por Microsoft Form (Cabezas et al., 2021)

Tabla 4. Pregunta 4 ¿Cuán satisfecho está con la disponibilidad de la información brindada por el docente (horarios de clase, tareas, etc.)?

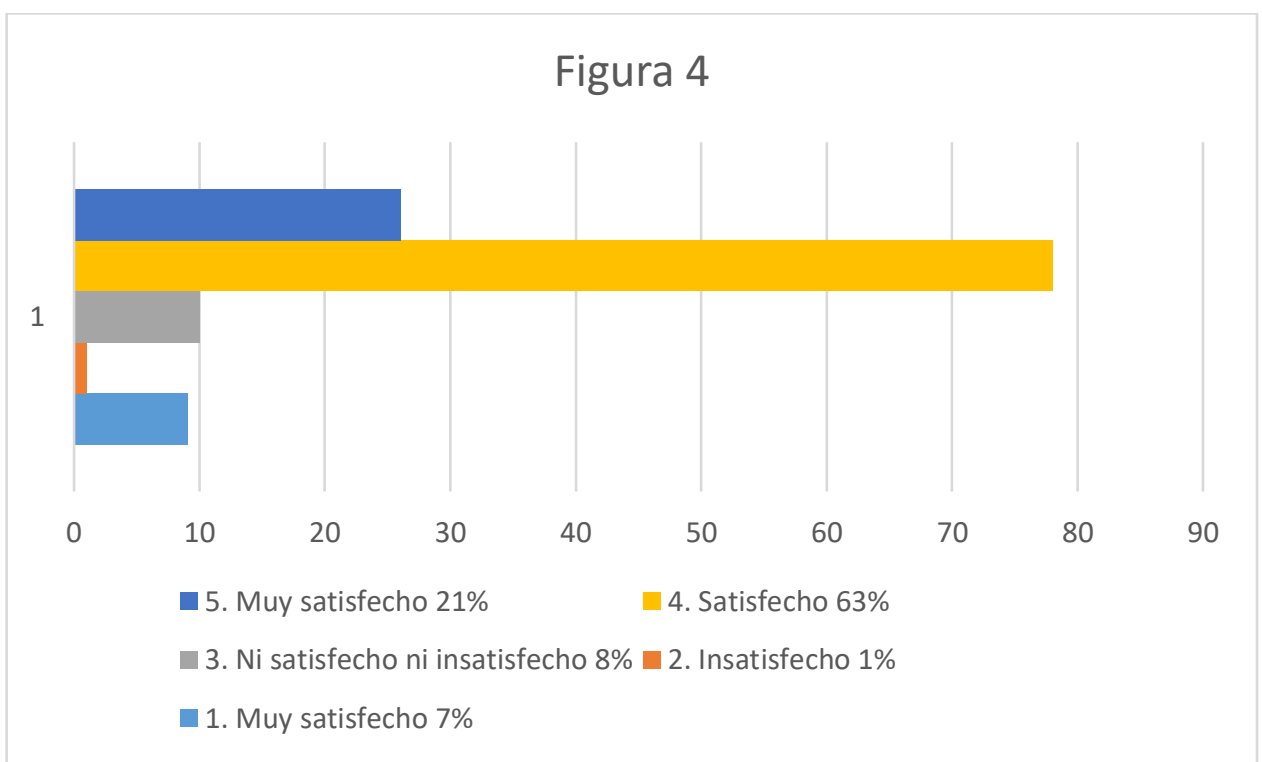

Fuente: Encuesta divulgada por Microsoft Form (Cabezas et al., 2021) 
Tabla 5. Pregunta 5 ¿Cuán satisfecho está con los métodos de enseñanza utilizados (principios y prácticas que el docente aplica en el entorno digital)?

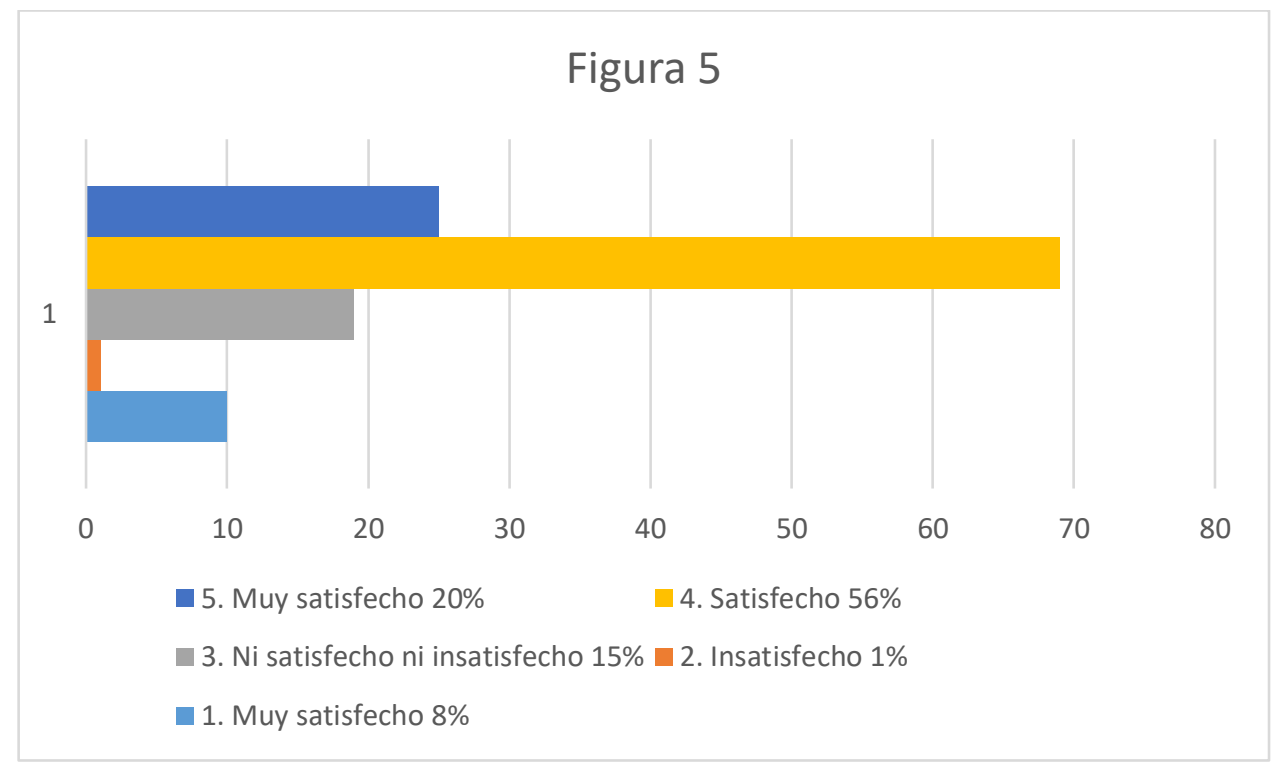

Fuente: Encuesta divulgada por Microsoft Form (Cabezas et al., 2021)

Tabla 6. Pregunta 6 ¿Qué tan satisfecho está con la retroalimentación del docente (en clase y en relación con las pruebas)?

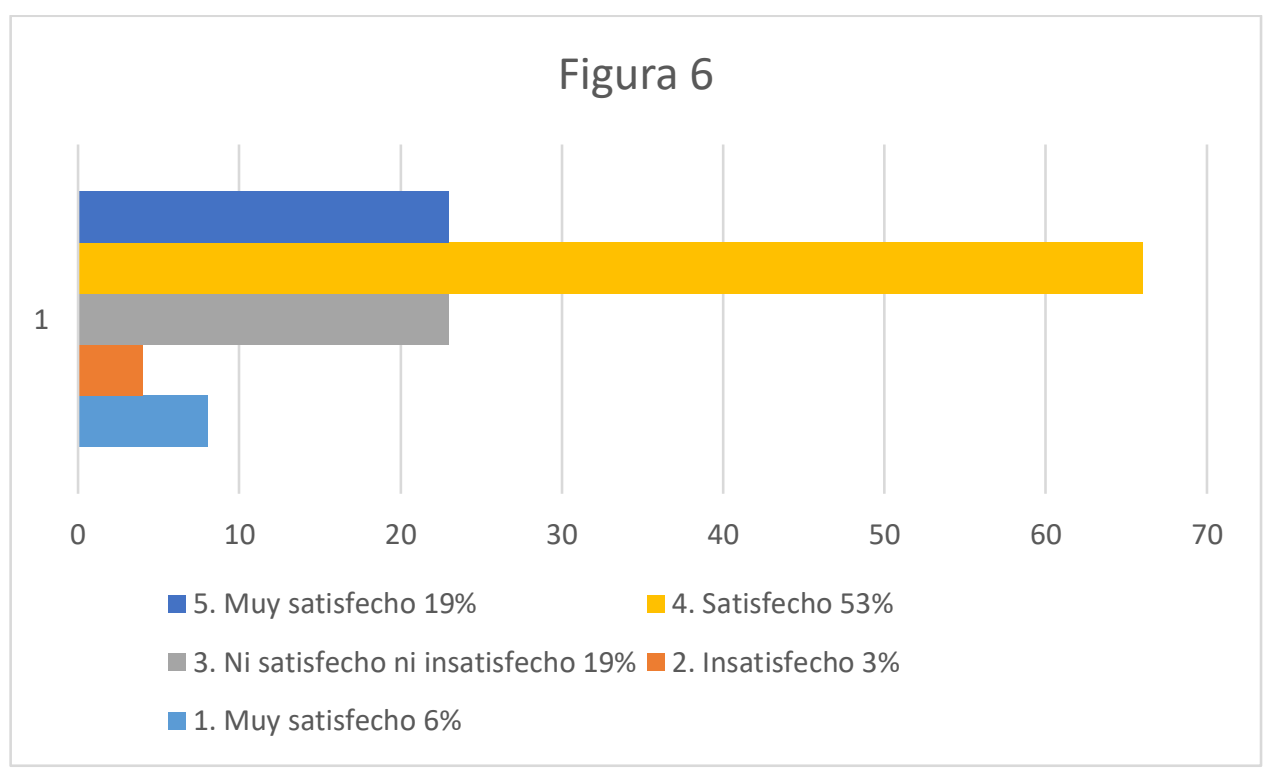

Fuente: Encuesta divulgada por Microsoft Form (Cabezas et al., 2021) 
Tabla 7. Pregunta 7 ¿Qué tan satisfecho está con la organización y aplicación de pruebas?

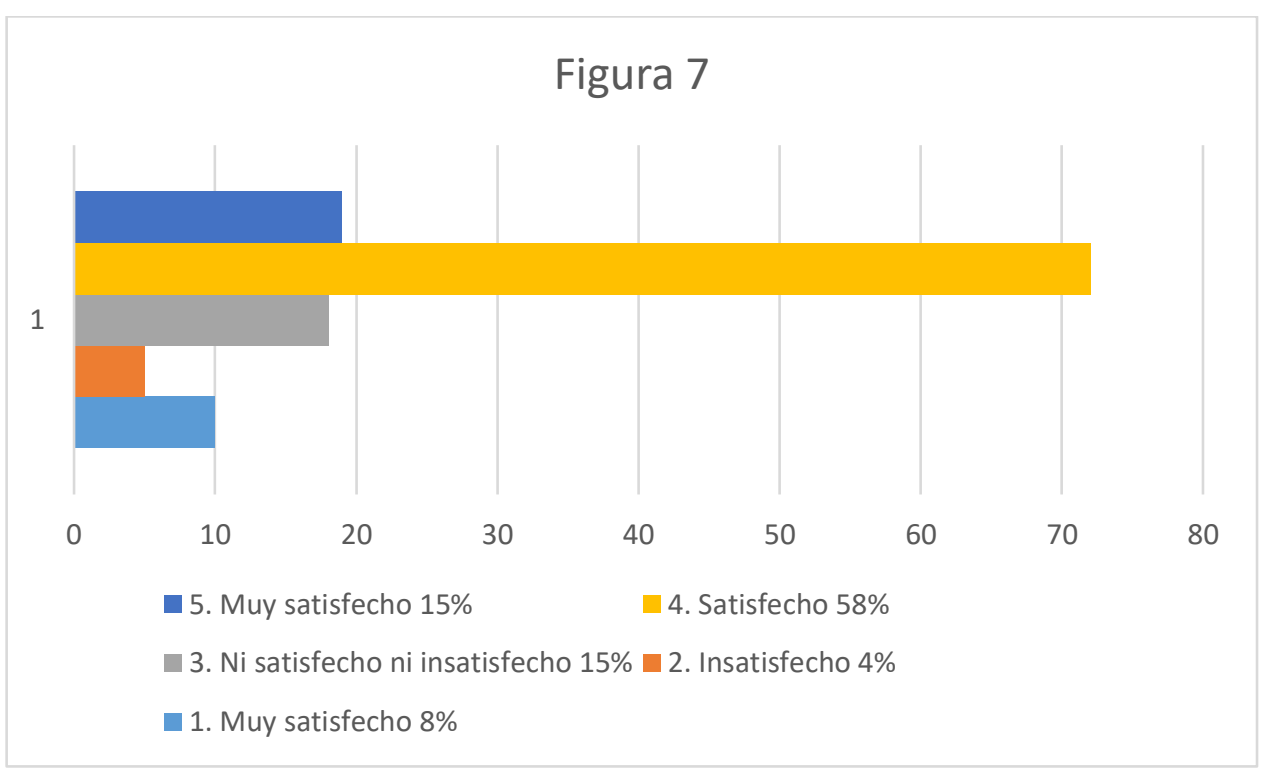

Fuente: Encuesta divulgada por Microsoft Form (Cabezas et al., 2021)

Tabla 8. Pregunta 8 ¿Qué tan satisfecho está con su desarrollo personal (académico y relacionado con el idioma)?

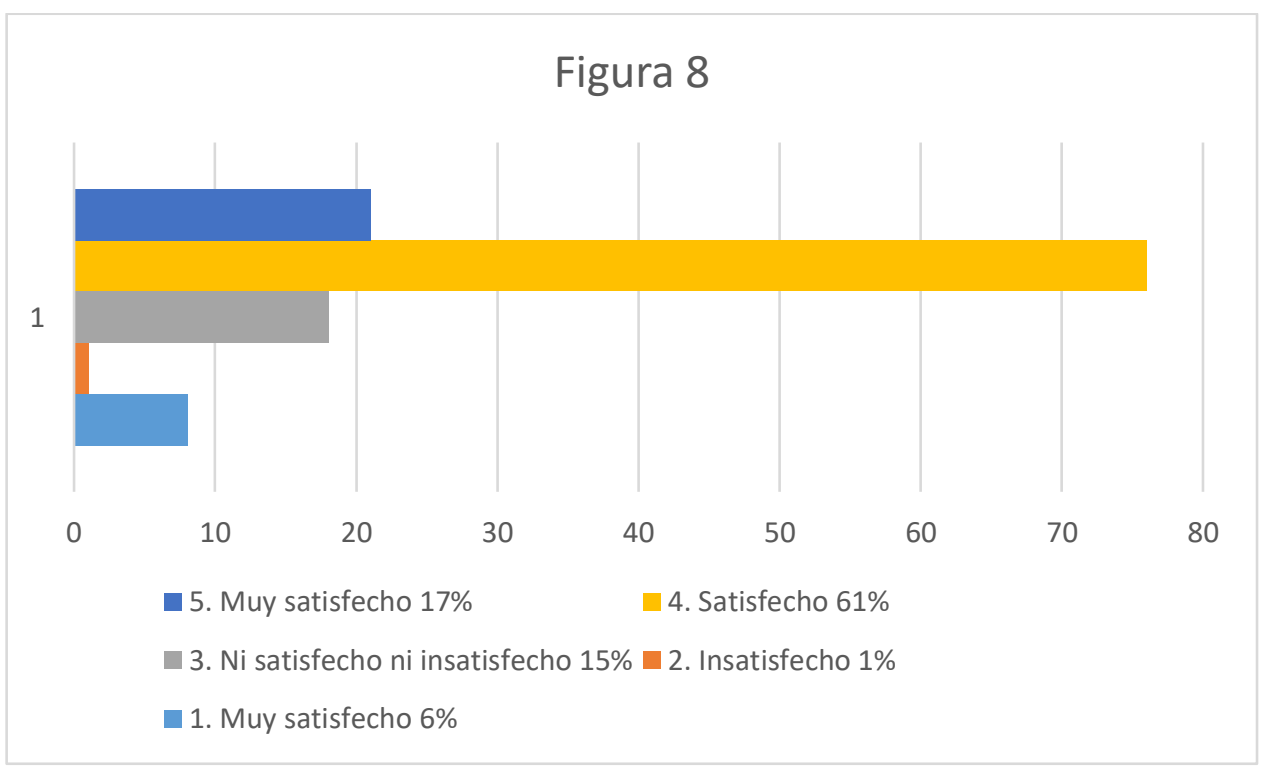

Fuente: Encuesta divulgada por Microsoft Form (Cabezas et al., 2021) 
Tabla 9. Pregunta 9 ¿Cómo describiría su experiencia general relacionada con la enseñanza y el aprendizaje en un entorno totalmente digital comparado con un entorno presencial?

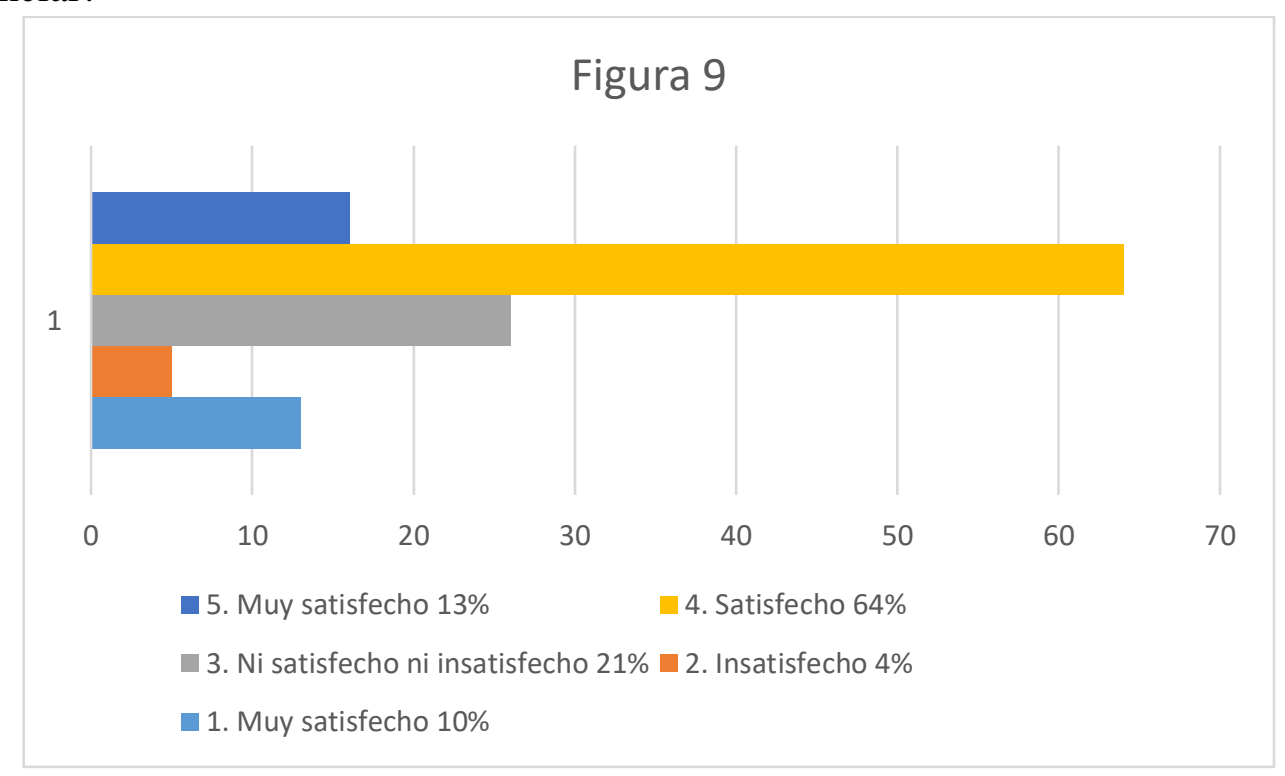

Fuente: Encuesta divulgada por Microsoft Form (Cabezas et al., 2021)

\section{Discusión}

Los niveles de satisfacción de los estudiantes son un indicador que permite el mejoramiento, el mantenimiento o cambio de las diferentes estrategias utilizadas a la hora de impartir clases en ámbitos educativos de cualquier nivel.

Debido a la pandemia la educación superior ha tenido un gran cambio con el implemento de clases totalmente virtuales. Por esta razón el objetivo de este estudio fue el de conocer los niveles de satisfacción de los estudiantes que toman la asignatura de inglés para valorar estos índices en un entorno educativo $100 \%$ online.

Se puede verificar en los resultados que existió un mayor porcentaje de elección para la opción 4 que correspondía a "satisfecho" en todas las preguntas por otro lado la opción 2 "insatisfecho" fue la menos escogida en todas las preguntas.

Un porcentaje del correspondiente a la opción 4 "satisfecho" para las preguntas 1 a 3 respectivamente no permite reconocer que la disponibilidad de materiales de aprendizaje, la dinámica en un entorno digital y la comunicación con el docente deja satisfechos a los estudiantes.

Cabe recalcar que los estudiantes son nativos digitales quienes están capacitados en su gran mayoría al entendimiento y uso de las herramientas virtuales para su desarrollo académico y personal. (García et al., 2007; Piscitelli, 2008) 
Por ello la brecha digital en el aspecto de familiaridad con la interfaz de trabajo no ha influido en gran medida para sus actividades escolares, sin embargo, existe una brecha digital en cuanto a la accesibilidad para estos medios. (Vivanco-Saraguro et al., 2020)

Estas respuestas coinciden con los datos obtenidos en el estudio de Prodanovic en donde se refleja una media de 3.91 quedando en una escala de satisfacción por parte de los estudiantes a las clases en un entorno virtual.(Prodanović \& Gavranović, 2021)

Estudios realizados por Cabezas y colaboradores manifiestan que no todos los estudiantes poseen las mismas condiciones para el acceso a las plataformas virtuales y de la misma manera en su conectividad por la carencia de dispositivos para este fin lo cual genera en el alumno la falta de interés y niveles bajos de satisfacción para las clases online. (Cabezas et al., 2021)

Estas afirmaciones pueden verse reflejadas en la elección de las opciones 12 y 3 de las preguntas 1-3 en este estudio ya que las mismas están relacionadas íntimamente con las herramientas digitales usadas por los alumnos y la conectividad de estos para poder acceder a sus clases.

Para las preguntas 4 a 7 que son referidas netamente a la apreciación sobre el docente y el manejo de sus competencias en la docencia. Se verifica que en estas preguntas la opción 4 fue la más elegida y la 2 la menos elegida.

Esto nos permite evidenciar el grado de satisfacción por parte de los alumnos hacia las actitudes, aptitudes y uso de herramientas digitales por parte del docente. La utilización de las TICs impacta de manera directa ya sea de manera positiva o negativa en esta modalidad en educación.(Herrera, 2015)

La generación de docentes antiguos debe estar consciente que son "inmigrantes digitales" por esta razón él o ella debe caracterizarse por la innovación, creatividad basada en la evidencia científica dirigiéndolo hacia su metodología de enseñanza. (García et al., 2007)

A pesar de que sean inmigrantes digitales se evidenció que los alumnos se encuentran satisfechos en cuanto a la comunicación, información brindada, métodos de enseñanza, retroalimentación y evaluación por parte del docente.

Estos datos coindicen con la investigación de Prodanovic en donde resalta que los beneficios de las herramientas digitales no puedes ser rechazadas y pone fin a las especulaciones que la mejor forma de enseñar y aprender un idioma extranjero es mediante una clase presencial. (Prodanović \& Gavranović, 2021)

En cuanto a la satisfacción personal y académica de los estudiantes se observó que eligieron en gran porcentaje la opción 4 de igual manera sucedió para el cuestionamiento relacionado a la comparativa con una educación presencial. 
Esto nos permite analizar que los estudiantes al tener adecuadas herramientas y accesibilidad a ellas pueden generar un conocimiento adecuado y además un interés por su desarrollo ya que se encuentra en su "hábitat natural de aprendizaje".

Si bien es cierto que no todos gozan de las mismas posibilidades se debe recalcar que si se le brinda al estudiante estas herramientas de conectividad ellos serán capaces de tener un nivel de satisfacción elevado además de poder gozar de otras actividades de forma simultánea en el día ya que no tiene inconvenientes de movilidad o gastos extras que supone una educación presencial. (Cabezas et al., 2021)

Los resultados de este estudio deberán ser bien analizados y entender su población de investigación ya que es una muestra reducida y que existió un único docente con experiencia en plataformas en línea y clases de inglés. Se exhorta que se amplíe el campo de investigación a una mayor cantidad de estudiantes y profesores con diferentes niveles de experiencia en este tipo de educación.

\section{Conclusiones.}

- Los niveles de satisfacción fueron altos para todas las interrogantes lo cual enaltece la educación virtual y se consolida como una alternativa educativa para la enseñanza y aprendizaje del idioma inglés como lengua extranjera. Se puede comprobar que no es necesario una educación presencial para satisfacer los niveles de satisfacción de los alumnos. Tanto los docentes como los alumnos deben estar relacionados con el entorno virtual para el aprovechamiento y satisfacción del proceso enseñanza-aprendizaje.

\section{Referencias bibliográficas.}

Alonso, A. M. (2010). Evaluación de la satisfacción del alumnado de cursos virtuales en la Empresa de Telecomunicaciones de Cuba, S.A. (ETECSA). Edutec: Revista Electrónica de Tecnología Educativa, $32,4$. http://dialnet.unirioja.es/servlet/articulo?codigo=3256490\&info=resumen\&idioma $=\mathrm{ENG}$

Cabezas, L. E., Moyota Amaguaya, P. P., \& Moyota Amaguaya, P. P. (2021). Conectividad y el uso de herramientas digitales en el aprendizaje del idioma inglés en estudiantes universitarios en tiempos del covid-19. ConcienciaDigital, 4(1.1), 307-326. https://doi.org/10.33262/concienciadigital.v4i1.1.1565

Flores, E. F., Cid, F. M., Briceño, M. H., Duarte, S. A. G., Silva-Salse, Á., Peña-Troncoso, S., Retamal, F. C., Flores, P. G., Cárcamo, F. P., Acevedo, V. B., Vera, G. Z., ZavalaCrichton, J. P., Sánchez, J. M., Brevis-Yéber, M., \& Olivos, C. L. (2020). Level of satisfaction of online classes by students of Physical Education of Chile in times of pandemic. Retos, 41, 123-130. https://doi.org/10.47197/RETOS.V0I41.82907

Garcia Bejarano, A., Angarita, J., \& Velandia Mesa, C. (2013). Implicaciones 
pedagógicas del uso de las TICs en la educación superior. Revista de Tecnología, 12(3), 36-56. https://doi.org/10.18270/rt.v12i3.1750

García, F., Portillo, J., Romo, J., \& Benito, M. (2007). Nativos digitales y modelos de aprendizaje. CEUR Workshop Proceedings, 318.

Herrera, A. M. (2015). Una mirada reflexiva sobre las TIC en educación superior. Revista Electronica de Investigacion Educativa, 17(1), 1-4.

Monasterio, D., \& Briceño, M. (2020). Educación mediada por las tecnologías: un desafío ante la coyuntura del covid-19. Observador Del Conocimiento, 5(1), 136-148.

Piscitelli, A. (2008). Nativos Digitales. Universidad de Lima - Contratexto, O(16), 43-56. https://revistas.ulima.edu.pe/index.php/contratexto/article/view/782

Prodanović, M., \& Gavranović, V. (2021). Students' satisfaction with learning experience in Covid-19 imposed virtual learning environment. Revista Publicando, 8(29), 124 131. https://doi.org/10.51528/rp.vol8.id2190

Santana-Sardi, G. A., Castro-Coello, R. L., Gutiérrez-Santana, J. A., \& Verónica Cecilia Zambrano-Palacios. (2020). La Educación Superior ecuatoriana en tiempo de la pandemia del Covid-19. 6, 757-775.

Vivanco-Saraguro, A., Internacional, C., Cáceres-muñoz, J., Martín-sánchez, M., Mexican, T., Tosso, M. P., Casado, C. M., Quiroz, C., Nacional, U., De México, A., Álvarez, M., Juárez Pérez-Cea, J., Oña Cots, J. M. de, Lorena, M., \& BonillaGuachamín, J. A. (2020). Desigualdades educativas y la brecha digital en tiempos de COVID-19. CienciAmérica, 9(2), 115-121. https://revistas.uam.es/riejs/article/view/12111

Zambrano R., J. (2016). Factores predictores de la satisfacción de estudiantes de cursos virtuales. RIED. Revista Iberoamericana de Educación a Distancia, 19(2), 217. https://doi.org/10.5944/ried.19.2.15112

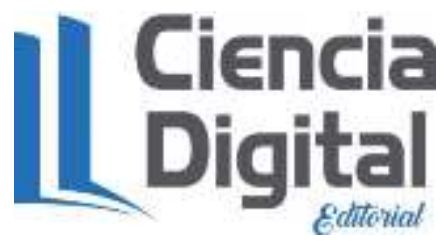




\section{PARA CITAR EL ARTÍCULO INDEXADO.}

Cabezas Arévalo, L. E. (2021). Nivel de satisfacción en el aprendizaje del idioma inglés en un entorno totalmente digital por tiempos de Covid-19. AlfaPublicaciones, 3(3.1), 116-128. https://doi.org/10.33262/ap.v3i3.1.81

\section{Ciencia \\ Digital \\ Edtorial}

El artículo que se publica es de exclusiva responsabilidad de los autores y no necesariamente reflejan el pensamiento de la Revista Alfa Publicaciones.

El artículo queda en propiedad de la revista y, por tanto, su publicación parcial y/o total en otro medio tiene que ser autorizado por el director de la Revista Alfa Publicaciones.
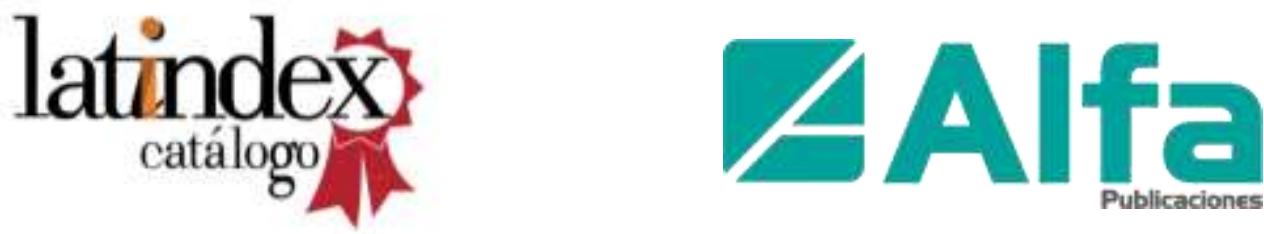